\title{
Why Do the Main Sequence Stars Have Similar Chemical Composition?
}

\author{
Stanislaw Halas ${ }^{1}$, Tomasz Durakiewicz ${ }^{2}$ \\ ${ }^{1}$ Institute of Physics, Maria Curie-Sklodowska University, Lublin, Poland \\ ${ }^{2}$ Los Alamos National Laboratory, MPA-CMMS Group, Los Alamos, USA \\ Email: stanislaw.halas@umcs.lublin.pl, tomasz@lanl.gov
}

Received December 6, 2011; revised January 17, 2012; accepted January 30, 2012

\begin{abstract}
In this short note we have reconsidered the Jeans criterion for gravitational contraction of a gas nebula at different temperatures, from the present-day background radiation temperature $(2.8 \mathrm{~K})$ to those which existed at the early stage of the Universe. We demonstrate that the initial mass of quasars cannot be of the order of single galaxy masses, but rather $10^{6}$ solar mass only. If they have larger masses, it must be the result of subsequent accretion process. Nevertheless quasars, formed prior to the stars, were the immediate source of the elements heavier than helium.
\end{abstract}

Keywords: Stars; Quasars; Background Radiation; Jeans Criterion; Gravitational Contraction; Nebulae

\section{Introduction}

The chemical composition of stars is related to the advancement of their evolution. Stars are born from various size interstellar nebulae and die as white dwarfs, neutron stars or black holes. During the latest evolutional stages a significant fraction of the star's mass is rejected back into space, what enriches the resources of interstellar matter.

According to the ideas of nucleosynthesis $[1,2]$ the younger stars should be more enriched in "metals", i.e. elements heavier than He. However, the observed metal abundances of the main sequence stars in the youngest open clusters and those in the oldest globular clusters appear to be very similar. Stars and clusters in our Galaxy show an excess of metals even in their age range of 5 - 9 billion years ( $\mathrm{Ga}$ ) [3]. Moreover, the $\mathrm{K}$ and $\mathrm{H}$ lines of the ionized calcium are most pronounced in the most distant galaxies and these (metallic) lines were used by Edwin P. Hubble for his redshift evaluation.

It is the purpose of this short note to explain this paradox on the basis of the Jeans criterion [4]. For the explanation we will return to the earliest stage of star formation, i.e. to gravitational contraction of a sufficiently large gaseous nebula.

\section{Theoretical Background}

First we will demonstrate that the mass of newly formed stellar object (from a gaseous cloud in space) depends on the density of the protogenic nebula and its temperature. Let us consider a uniform spherical nebula of radius $R$ containing $N$ atoms of mass $m$ each. Such an object be- comes unstable when its gravitational energy exceeds thermal energy. The initial thermal energy, prior to gravitational contraction, solely depends on the space temperature, $T$, which is equal to the background radiation temperature, if the considered nebula is sufficiently distant from any hot object. Hence in this case for a low temperature gas being composed of single atoms we have:

$$
E_{t}=\frac{3}{2} \cdot N k_{B} T,
$$

where $k_{B}$ is the Boltzmann constant. On the other hand the initial gravitational energy of the considered nebula is:

$$
E_{g}=\frac{3}{5} \cdot \frac{G M^{2}}{R}
$$

where $M$ is its total mass and $G$ is gravitation constant.

The nebula starts to contract if $E_{g}$ exceeds $E_{t}$, hence from the equality $E_{g}=E_{t}$, which the basis of the Jeans criterion, we can determine critical radius of the nebula and subsequently its mass. Substituting for the total mass of the nebula $N m=4 / 3 \pi R^{3} \rho$, where $\rho=n m$ is the gas density and $n$ is the number of atoms per unit volume, the following equation for the critical size of the nebula is obtained:

$$
R_{c}=\left(15 k_{B} T\right)^{1 / 2}\left(8 \pi G m^{2} n\right)^{-1 / 2}
$$

Substituting the numerical values into above equation, one obtains $R_{c}=2.11 \times 10^{20} \mathrm{~m} \cdot(T / n)^{1 / 2}$ or $R_{c}=6.83 \mathrm{pc} \cdot(T / n)^{1 / 2}$, where $T$ is expressed in kelvins and $n$ in $\mathrm{cm}^{-3}$. Having the initial radius of a nebula, we can calculate the mass of the star being formed, $M=$ 
$4 / 3 \pi R_{c}^{3} n m$. Substituting the mass of hydrogen atom for $m$, we obtain:

$$
M=6.49 \times 10^{31} \mathrm{~kg} \cdot T^{3 / 2} n^{-1 / 2},
$$

where $T$ is expressed in kelvins and $n$ in $\mathrm{cm}^{-3}$. This formula can be rewritten with solar mass units:

$$
M=32.6 M_{\odot} T^{3 / 2} n^{-1 / 2}
$$

Substituting into formula (4a) the present-day background temperature $(2.8 \mathrm{~K})$ and 100 hydrogen atoms per $\mathrm{cm}^{3}$ for $n$, we obtain $15.3 M \odot$.

This estimation is sensible, since the observed star masses fall into the range from 0.1 to $120 M_{\odot}$. Such density is observed in spiral arms of galaxies. For a roughly estimated average density of the present-day Universe $(n$ $=1 \mathrm{H}$ atom $/ \mathrm{cm}^{3}$ ) we obtain $153 M_{\odot}$, which is just above the upper limit of the observed masses. If such a massive star would be formed, then its life would be too short to be observed. On the other hand the nebulae in galaxies are strongly perturbed by the surrounding massive bodies, which often lead to the formation of a cluster instead of a single massive star [5].

Actually in the space of the present-day Universe any nebula contains not only hydrogen atoms, but also helium, carbon and atoms of heavier elements and many molecular species like $\mathrm{H}_{2}, \mathrm{H}_{2} \mathrm{O}, \mathrm{CH}_{4}, \mathrm{HCN}$, etc. These heavier components do not contribute as much as the hydrogen atoms to the pressure inside the nebula and to the total kinetic energy at the temperature of $2.8 \mathrm{~K}$, because the molecules are predominantly adsorbed on the clusters of $\mathrm{C}, \mathrm{Si}$ and metal atoms and altogether they form the cosmic dust particles. However, these molecular species and dust particles contribute to the gravitational energy, which depends on the total mass of the nebula according to Equation (2). Therefore the Jeans criterion should be modified with respect to the nebula composition. This is not a trivial task, but it is obvious that the masses obtained using formula (4a) are overestimated. Clearly, dusty nebulae may form into objects with much smaller mass than estimated above, e.g. small-mass stars of late spectral types and Jupiter-like giant planets.

\section{Early Universe}

It is not the purpose of this section to repeat the model of temperature and density evolution of the Universe from the time of a billion years ago $(1 \mathrm{Ga})$ to the Big Bang ( $14 \mathrm{Ga}$ before present). The literature on this subject is already abundant, see e.g. [6] and references therein. Going back in time by observing more and more distant objects, one notes that at certain time the super-giant quasi stellar objects (quasars) were formed rather than stars.

In order to obtain with formula (4) a mass of a single galaxy $\left(10^{10} M_{\odot}\right)$ instead of a single star, the expression $T^{3 / 2} n^{-1 / 2}$ has to attain a value of the order of $10^{9}$. This implies that temperature has to be at least $10^{6} \mathrm{~K}$. At such a high temperature the matter is not only completely ionized, but the thermonuclear $\mathrm{p}-\mathrm{p}$ cycle starts, which may prevent the gravitational contraction of a nebula, in spite of its size. In conclusion, the initial masses of quasars were lower than the mass of a typical galaxy. If they really attained such large masses, it must have been due to the subsequent accretion.

At temperatures below $10^{4} \mathrm{~K}\left(k_{B} T=1 \mathrm{eV}\right)$ hydrogen atoms are predominantly neutral and the quasi stellar objects formed at that time could attain masses of order $10^{6} \mathrm{M} \odot$. These objects were extremely explosive injecting newly formed heavy elements to the primordial hydrogen matter. For this reason even the oldest stars existing now in globular clusters were formed from hydrogen enriched in "metals" generated at the earliest stage of existence of the Universe.

\section{Conclusions}

A critical discussion of Jeans criterion for gravitational contraction of a gas nebula leads to conclusion that a major fraction of "metals" was formed in very early stage of evolution of the Universe. This answers the question raised in the title of this paper.

Moreover, the Jeans criterion leads to a reliable estimation of the upper limit of the initial masses of quasars. Quasars cannot attain the mass of a typical galaxy $\left(10^{10} M_{\odot}\right)$, but most likely they were formed at temperatures below $10^{4} \mathrm{~K}$ and their initial mass did not exceed $10^{6} M_{\odot}$. If they attained larger masses, it must have been a result of the subsequent accretion processes.

\section{Acknowledgements}

Authors thank Dr. P. Wozniak for critical reading of the manuscript and helpful comments.

\section{REFERENCES}

[1] E. M. Burbidge, G. R. Burbidge, W. A. Fowler and F. Hoyle, "Synthesis of the Elements in Stars," Reviews of Modern Physics, Vol. 29, No. 4, 1957, pp. 547-650. doi:10.1103/RevModPhys.29.547

[2] R. Q. Huang and K. N. Yu, "Stellar Astrophysics," Springer-Verlag, Singapore, 1998.

[3] G. Carro, Y. K. Ng and L. Portinari, "On the Galactic Disc Age Metallicity Relation," Monthly Notices of the Royal Astronomical Society, Vol. 296, No. 4, 1998, pp. 1045-1056. doi:10.1046/j.1365-8711.1998.01460.x

[4] J. Jeans, “Astronomy and Cosmology," Cambridge University Press, Cambridge, Republished 1961.

[5] J. M. Pasachoff and M. L. Kutner, "University Astronomy,” W.B. Saunders Co., Philadelphia, 1978.

[6] M. Demiański, "Relativistic Astrophysics (in Polish)," Chapter 10, PWN Warszawa, 1991. 